\title{
microRNA-320a inhibits tumor invasion by targeting neuropilin 1 and is associated with liver metastasis in colorectal cancer
}

\author{
YUJUN ZHANG $^{1}$, XIANGJUN HE ${ }^{1}$, YULAN LIU ${ }^{1,2}$, YINGJIANG YE $^{3}$, HUI ZHANG $^{3}$, \\ PEIYING HE ${ }^{1}$, QI ZHANG ${ }^{1}$, LINGYI DONG ${ }^{3}$, YUJING LIU $^{1}$ and JIANQIANG DONG ${ }^{1}$ \\ ${ }^{1}$ Institute of Clinical Molecular Biology, and Departments of ${ }^{2}$ Gastroenterology and ${ }^{3}$ General Surgery, \\ Peking University, People's Hospital, Beijing 100044, P.R. China
}

Received September 13, 2011; Accepted October 26, 2011

DOI: 10.3892/or.2011.1561

\begin{abstract}
MicroRNAs (miRNAs) have been implicated in regulating diverse cellular pathways. Although there is emerging evidence that various miRNAs function as oncogenes or tumor suppressors in colorectal cancer (CRC), the role of miRNAs in mediating liver metastasis remains unexplored. The expression profile of miRNAs in liver metastasis and primary CRC tissues was analyzed by miRNA microarrays and verified by real-time polymerase chain reaction (PCR). In $62 \mathrm{CRC}$ patients, the expression levels of miR-320a were determined by real-time PCR, and the effects on migration and invasion of miR-320a were determined using a transwell assay. miR-320a target genes were confirmed by luciferase assay, real-time PCR and Western blot analysis. A set of miRNAs was found to be dysregulated in the liver metastasis tissues compared to matched primary CRC tissues, and the expression levels of miR-320a were significantly decreased in the liver metastasis tissues examined. miR-320a was correlated with tumor progression in CRC. miR-320a was downregulated in liver metastatic colon cancer cells and inhibited liver metastatic colon cancer cell migration and invasion. miR-320a directly binds to the 3'UTR of neuropilin 1 (NRP-1), a protein that functions as a co-receptor of vascular epithelial growth factor. miR-320a downregulated the expression of NRP-1 at both the mRNA and protein levels. These data demonstrated that miR-320a may be useful for identifying CRC patients that are at an elevated risk for developing liver metastasis. Our findings suggest that miR-320a may be a novel therapeutic candidate for the treatment of colorectal cancer.
\end{abstract}

Correspondence to: Dr Yujun Zhang or Dr Yulan Liu, Institute of Clinical Molecular Biology, Peking University, Beijing 100044, P.R. China

E-mail: yujun_zhang66@yahoo.com.cn

E-mail: liuyulan@pkuph.edu.cn

Key words: microRNAs, colorectal cancer, liver metastasis, neuropilin 1

\section{Introduction}

Colorectal cancer (CRC) is the second most common cause of cancer-related death worldwide (1). Approximately $50 \%$ of patients diagnosed with CRC die as a result of complications from distant metastases, which occur mainly in the liver. The occurrence of liver metastasis ranges from $20 \%$ in stage II to $70 \%$ in stage IV CRC patients, according to the UICC stage guidelines. Of all patients who die of advanced colorectal cancer (ACRC), 60-70\% display liver metastasis (2). Metastasis to the liver is the major cause of death in CRC patients (3). The formation of liver metastases is a multistep process involving the escape of tumor cells from a primary CRC, intravasation into the systemic circulation, survival during transit in the vasculature, extravasation into the parenchyma of liver tissues, and ultimately the outgrowth of macroscopic liver metastasis formation through proliferation and angiogenesis (4-6). Although research has identified multiple genes responsible for liver metastasis (7), the epigenetic alterations that drive metastasis to the liver are largely unknown.

MicroRNAs (miRNAs) are a family of small, highly conserved non-coding RNAs that post-transcriptionally regulate gene expression through sequence-specific interactions with the 3'untranslated regions (UTRs) of cognate mRNA targets. miRNAs silence genes by promoting both translational inhibition and mRNA degradation (8). More than 1000 human miRNAs have currently been identified, and each individual miRNA may control hundreds of target genes. Approximately $30 \%$ of human genes are believed to be putative miRNA targets (9). More than 50\% of miRNA-encoding loci are located within chromosomal regions that are altered during tumorigenesis (10). Some miRNAs can function either as oncogenes or tumor suppressors $(11,12)$. Expression profiling analyses have revealed characteristic miRNA signatures in certain types of human cancers $(13,14)$.

MiRNAs contribute to the pathogenesis of CRC by regulating the expression of oncogenes and tumor suppressors and also by directly functioning as oncogenes or tumor suppressors $(15,16)$. The role of miRNAs in the development of metastasis has been demonstrated in several cancer types, including breast cancer, hepatocellular carcinomas, head and neck cancer and brain tumors (17). Most studies involving CRC have analyzed the association between miRNAs and 
metastasis using both in vitro and mouse models. Most tissuebased experiments derived from CRC patients are only able to identify differential miRNAs using primary tumor tissues versus normal tissue (15). Currently, only two studies have compared primary tumors to metastatic tissues using miRNA expression profiles. One of these studies used an unmatchedpair approach (18). This type of unmatched comparison is uncertain due to the differences in endogenous miRNA levels that are observed in different primary colorectal carcinoma samples. The other study used miRNA microarray analysis to compare 10 primary colon tumors with one related metastatic lymph node. This analysis identified 21 miRNAs that were differentially expressed between the primary and metastatic tumors (19). The expression of miRNAs is highly tumor- and tissue-specific, highlighting the need for more information on the association between miRNAs and liver metastases in colorectal carcinomas.

In this study, we identified miRNAs, including miR-320a, that were correlated with liver metastasis by comparing miRNA expression patterns in primary carcinomas and matched liver metastatic tissue. miR-320 expression levels were found to be downregulated in primary breast cancer (BC) (20) and intrahepatic cholangiocarcinoma (21). miR-320 expression also inhibited HL-60 cell proliferation by targeting transferrin receptor 1 (CD71) (22). These data suggest that miR-320 may play an important role in the development of cancer.

We further demonstrated that the downregulation of miR-320a was associated with tumor progression and the development of liver metastasis. Enhanced expression of miR-320a suppressed the invasion and migration of the liver metastatic colon cancer LoVo cell line in vitro. Using bioinformatics-based analysis and luciferase assays, we identified neuropilin 1 (NRP-1) as a putative miR-320a target that may be involved in the promotion of cancer cell migration, invasion and angiogenesis. These results suggest a novel function for miR-320a as a crucial modulator of liver metastasis in CRC. Our findings indicate that miR-320a may be a potential target for diagnosis and therapy of CRC.

\section{Materials and methods}

Patients. In this study, samples of primary CRC, normal adjacent tumor tissue, and liver metastasis tissues were collected from 62 patients treated at People's Hospital at Peking University between 2002 and 2009. The group consisted of 22 women and 40 men, with a median age of 66 years (range, 41-89). Distribution according to clinical stage (International Union Against Cancer staging system) was as follows: stage I in 4 cases, stage II in 21, stage III in 20 and stage IV in 17 cases. Seventy-five tissue samples were collected from surgically resected tissues, of which 49 samples were taken from primary tumors, 6 matched pairs were derived from adjacent non-tumor mucosal tissues and primary tissues, and 7 matched pairs were taken from both liver metastasis tissues and primary tissues. All samples were immediately stored in liquid nitrogen. Patients treated with radiotherapy or chemotherapy prior to surgery were not included in this study. The study protocol conformed to the guidelines of the ethics committee. It was approved by the Review Board of People's Hospital at Peking University.
Cell culture. The human colon cancer cell lines SW480, SW620 and LoVo were purchased from the Cell Bank of China Union Medical University. SW480 and LoVo were incubated at $37^{\circ} \mathrm{C}$ in a $10 \% \mathrm{CO}_{2}$ atmosphere and maintained in Dulbecco's modified Eagle's medium (DMEM) supplemented with $10 \%$ fetal bovine serum and $2 \mathrm{mM}$ L-glutamine (Invitrogen, Carlsbad, CA). SW620 cells were maintained in L-15 medium supplemented with $10 \%$ fetal bovine serum. HT-29 cells were purchased from Shanghai Cell Bank and cultured in DMEM supplemented with $10 \%$ fetal bovine serum.

miRNA microarray. For miRNA microarray analyses, total RNA was harvested from four pairs of colorectal carcinoma and matched liver metastasis tissues using TRIzol (Invitrogen) and purified using the RNeasy Mini kit (Qiagen, Germany) according to the manufacturer's instructions. The concentration and quality of total RNA were measured using a NanoDrop ND-1000 at 260 and $280 \mathrm{~nm}$ (A260/280) and confirmed by gel electrophoresis. Each RNA sample was labeled using the miRCURY ${ }^{\mathrm{TM}} \mathrm{Hy} 3^{\mathrm{TM}} / \mathrm{Hy}^{\mathrm{TM}}$ Power Labeling kit and hybridized on the miRCURY TM LNA array (v.11.0) (Exiqon, Denmark). This array contains probes for 1700 mature miRNAs. Scanning was performed using the Axon GenePix 4000B microarray scanner (Axon Instruments, Union City, CA). GenePix pro V6.0 was used to interpret the raw intensity of the image. The intensity of the green signal was calculated after background subtraction and four replicate spots from each probe on the same slide were averaged. We used the median normalization method to obtain normalized data. Normalized data $=($ foreground - background $) /$ median, where the median is the 50 percentile of microRNA intensity. This value was $>50$ in all samples after background correction. Unsupervised hierarchical clustering was performed on the microarray data. Only those miRNAs with a $>1.5$-fold increase or 1.5-fold decrease in expression in two matched samples were considered significantly changed.

RNA isolation and reverse transcription. Total RNA isolation and small RNA enrichment procedures were performed using the miRNeasy RNA Isolation kit (Qiagen, USA) according to the manufacturer's instructions. RNA concentration and purity were both controlled using the Nanodrop ND 1000. RNA integrity was verified by $1 \%$ agarose gel electrophoresis, and small RNA was analyzed using 3.5\% NuSieve agarose electrophoresis (Cambrex, USA). RNA $(1 \mu \mathrm{g})$ was polyadenylated using the Poly(A) Tailing kit (Ambion, USA) in accordance with the manufacturer's instructions. RNAs were dissolved in diethylpyrocarbonate (DEPC)-treated water and reversetranscribed using SuperScript ${ }^{\mathrm{TM}}$ II Reverse Transcriptase (Invitrogen) and an anchored oligo dT RT primer with a 40-nt extension at the 5' end (5'-GCT GTC AAC GAT ACG CTA CGT AAC GGC ATG ACA GTG(T), VN-3' where $\mathrm{V}=\mathrm{A}, \mathrm{G}$, or $\mathrm{C}$ and $\mathrm{N}=\mathrm{A}, \mathrm{G}, \mathrm{C}$, or $\mathrm{T}$ ).

Real-time PCR. Real-time PCR was performed using the SYBR-Green Real-time PCR Master Mix kit (Toyobo, Osaka, Japan) and the Opticon 2 (Bio-Rad Laboratories, Hercules, CA, USA) with gradient temperature function. A forward primer specific to each miRNA and a common reverse primer were used for the amplification. Raising annealing temperatures 
$12-14{ }^{\circ} \mathrm{C}$ above the $\mathrm{Tm}$ of the primer resulted in a maximal increase in amplification specificity without decreasing sensitivity. This allowed for the differential expression analysis of miRNAs having as few as one nucleotide sequence difference. For each PCR assay, $2 \mu \mathrm{l}$ of template cDNA (equivalent to a $\sim 1 \mathrm{ng}$ small RNA) was mixed with $10 \mu \mathrm{l}$ of $2 \mathrm{X}$ SYBR-Green PCR master mix and $200 \mathrm{nmol} / \mathrm{l}$ each of the forward and reverse primers at a final volume of $20 \mu \mathrm{l}$. For low-expression RNAs, the concentration of template cDNA was $\sim 10 \mathrm{ng}$. The amplification protocol consisted of $20 \mathrm{sec}$ of denaturation at $94^{\circ} \mathrm{C}, 20 \mathrm{sec}$ of annealing at $55-70^{\circ} \mathrm{C}$ for each specific miRNA primer, and $30 \mathrm{sec}$ of extension at $70^{\circ} \mathrm{C}$ for 40 cycles.

All reactions for each sample were performed in triplicate. The PCR results were analyzed using Opticon Monitor 3 software (Bio-Rad Laboratories). Based on the threshold cycle $(\mathrm{Ct})$, the quantity of each miRNA relative to a reference gene was calculated using the formula $2^{-\Delta \mathrm{CT}}$ automatically, where $\Delta \mathrm{CT}=\left(\mathrm{CT}_{\text {miRNA }}-\mathrm{CT}_{\text {reference RNA }}\right)$ and $\Delta \Delta \mathrm{CT}=\left(\Delta \mathrm{CT}_{\text {metastasis }}\right.$ $\left.\Delta \mathrm{CT}_{\text {control }}\right)$. The formula $2^{-\Delta \Delta \mathrm{CT}}$ was calculated to demonstrate the relative level of miRNA expression compared to the negative control.

Primer design. Human microRNA sequences were selected and blasted against the miRBase (http://microma.sanger. ae.uk). Sequences (AAA AAA AAA AAA AAAAAA AAA AAA CAC TGT CAT GCC GTT ACG TAGCGT ATC GT GAC AGC) were added to the end of each microRNA. Primer 3 (http://fodo.wi.mit.edu/) was used to select upstream primers that possess the sequences described above. Primers for similar microRNAs can produce non-specific amplification; thus, primers containing variant bases were designed at the 3 ' termini of the microRNA sequence based on the results of the microRNA BLAST analysis. The first 20 nucleotides located at the $5^{\prime}$ end of the RT primer were defined as the common reverse primer (5'-GCTCTC AAC GAT ACG CTA CG-3') (23-25).

Selection of candidate endogenous control genes for normalization. Normalization is an essential step for the accurate quantification of miRNA levels. Ten miRNAs were selected from our miRNA array expression profiling data based on their modified $\mathrm{z}$-score ranking (26). We evaluated the stability of 10 candidate miRNAs and 5S using GeNorm (27) and NormFinder (28) in 10 colorectal tumor tissues. The ranking of the candidates was determined using these software programs.

Lentivirus production and infection. Human genomic fragments of miR-320a precursors along with flanking regions of $\sim 300$ bp were amplified by PCR using human genomic DNA as a template. PCR primers containing the XhoI and BamHI restriction sites were designed (forward primer: HSA-MIR320A-XhoI, CAACAGAAGGCTCGAGGGAAGTCTGC GTGGCAGG, reverse primer: HSA-MIR-320A-BamHI, ATTCTGATCAGGATCCGAGGCGAATCCTCACATTG).

The PCR products were cloned into the $\mathrm{pGIPZ}$ vector (Open Biosystems) to generate pGIPZ-320a. Sequencing was used to screen and identify the accuracy of construct insertion. The pGIPZ-NC vector was used as a negative control. Lentivirus was packaged in HEK 293 T cells after the co-transfection of
$252 \mu \mathrm{g}$ of the pGIPZ-320a vector with $168 \mu \mathrm{g}$ of the packaging plasmid vector PCD/NL-BH*DDD (Addgene) and $84 \mu \mathrm{g}$ of the envelope plasmid PLTR-GvVector (Addgene). Viruses were harvested $48 \mathrm{~h}$ following infection. Virus titer $\left(1 \times 10^{9} / \mathrm{ml}\right)$ was determined using a dilution counting assay. LoVo cells were seeded into 6-well plates and transfected with lentivirus at a multiplicity of infection value of $50(\mathrm{MOI}=50)$ in the presence of $6 \mathrm{mg} / \mathrm{ml}$ Polybrene (GeneChem). LoVo cells were plated for migration and invasion assays at $24 \mathrm{~h}$ after infection.

Oligonucleotide transfection. miR-320a inhibitor and negative control oligonucleotides were chemosynthesized (Shanghai GenePhama Co., Ltd.). The oligonucleotides used in these studies were the miR-320a inhibitor (5'-UCGCCCUCUC AACCCAGCUUUU-3') and the miRNA inhibitor negative control (5'-CAGUACUUUUGUGUAGUACAA-3'). SW480 cells in each well of a 6-well plate were transfected with $200 \mathrm{nM}$ of the indicated oligonucleotide using Oligofectamine reagent (Invitrogen). SW480 cells were plated for migration and invasion assays at $48 \mathrm{~h}$ after transfection.

Luciferase reporter assay. A dual-luciferase reporter assay was performed to investigate whether NRP-1 expression is regulated by miR-320a. The 3'UTR of NRP-1, which contains a miR-320a binding site, was amplified by PCR. The PCR primers were designed to contain a HindIII restriction site (NRP1-3UTR-HindIII-forward, TTAAACAGTTAAGCTT AGGCAGACAGAGATGAAAAGAC; NRP1-3UTR-Hind III-reverse, ATCCTTTATTAAGCTTCCACTTCCCTTG TGTGACTC). As a negative control, mutations were generated in the NRP-1 3'-UTR miRNA binding site sequence (CAGCTTT $\rightarrow$ TCTTGAC) using a QuickChange SiteDirected Mutagenesis kit (Stratagene). PCR products were cloned into the luciferase reporter pMIR-REPORT vector (Ambion). All constructs were verified by sequencing. LoVo cells were seeded into 24-well plates and co-transfected with control and luciferase reporters using Lipofectamine ${ }^{\mathrm{TM}} 2000$ (Invitrogen) following the manufacturer's instructions. Firefly and Renilla luciferase activity was measured using the dual luciferase reporter assay system (Promega) after $48 \mathrm{~h}$ of incubation.

Cellular proliferation assays. To evaluate the effect of miR$320 \mathrm{a}$ on cellular proliferation, $2 \times 10^{3}$ LoVo cells infected with pGIF-320a and/or control vector were seeded into 96-well plates in complete medium. After 2 days, the cell viability was measured by MTT assay using the Cell Proliferation Kit I according to the manufacturer's instructions (Roche). Six replicates were performed for each condition.

Cell migration and invasion assays. The effects of miR$320 \mathrm{a}$ on cell migration and invasion were determined using 8- $\mu \mathrm{m}$ transwell filters (Costar, Corning, NY). For transwell migration assays, $2.5 \times 10^{4}$ LoVo or SW480 cells were plated in the top chamber containing a non-coated membrane. For invasion assays, $2 \times 10^{5}$ LoVo or SW480 cells were plated in the top chamber containing a Matrigel-coated membrane. The upper compartment was coated with $100 \mu 1$ Matrigel (BD Biosciences, San Jose, CA) for $2 \mathrm{~h}$ to form a matrix barrier. A suspension of cells in basal medium was added to the 
Table I. Fold-change of the miRNAs in primary CRC and liver metastasis tissues detected by miRNA microarray.

\begin{tabular}{|c|c|c|c|c|}
\hline \multirow[b]{2}{*}{ Gene name } & \multirow{2}{*}{$\begin{array}{c}\text { Fold-change } \\
\text { Metastasis/primary }\end{array}$} & \multicolumn{2}{|c|}{ Normalized } & \multirow[b]{2}{*}{ Regulation } \\
\hline & & Primary & Metastasis & \\
\hline hsa-miR-200b & 2.38 & 0.23 & 0.55 & Upregulated \\
\hline hsa-miRPlus-E1191 & 3.77 & 12.14 & 45.70 & Upregulated \\
\hline hsa-miRPlus-E1290 & 2.89 & 0.91 & 2.62 & Upregulated \\
\hline hsa-miR-106a & 1.65 & 0.96 & 1.58 & Upregulated \\
\hline hsa-miR-106b* & 2.32 & 0.36 & 0.84 & Upregulated \\
\hline hsa-miR-1301 & 1.55 & 0.31 & 0.49 & Upregulated \\
\hline hsa-miR-320a & 0.21 & 1.39 & 0.29 & Downregulated \\
\hline hsa-miRPlus-D1036 & 0.63 & 22.30 & 14.16 & Downregulated \\
\hline hsa-miR-200c & 0.60 & 2.12 & 1.28 & Downregulated \\
\hline hsa-miRPlus-E1252 & 0.44 & 12.39 & 5.42 & Downregulated \\
\hline hsa-miR-141 & 0.32 & 2.86 & 0.92 & Downregulated \\
\hline hsa-miR-1279 & 0.25 & 0.84 & 0.21 & Downregulated \\
\hline hsa-miR-320b & 0.47 & 1.15 & 0.54 & Downregulated \\
\hline
\end{tabular}

upper compartment. The lower compartment was filled with $500 \mu \mathrm{l}$ of basal medium containing $10 \%$ fetal bovine serum as a chemoattractant. The cells were incubated at $37^{\circ} \mathrm{C}$ in a $5 \% \mathrm{CO}_{2}$ humidified incubator for $24 \mathrm{~h}$. The non-migratory cells located on the upper surface were removed using a cotton swab, and the cells on the lower surface were fixed and stained with crystal violet. Cells that had migrated or invaded into each transwell filter were counted from ten randomly chosen fields in each well. Imaging and cell counting were performed at x100 magnification under an inverted microscope (Leica Corp., Germany). The experiment was performed in quadruplicate and repeated at least three times independently.

Western blot analysis. Cells or tissues were lysed in RIPA buffer [50 mmol/1 Tris-HCl, pH 8.0, $150 \mathrm{mmol} / 1 \mathrm{NaCl}$, $1 \%$ (vol/vol) Nonidet P-40, $0.5 \%$ (wt/vol) sodium desoxycholate, $0.1 \%$ (wt/vol) SDS] and protease inhibitors. After centrifugation at $14,000 \mathrm{rpm}$ for $15 \mathrm{~min}$, the supernatants were collected. Protein samples (15 $\mu \mathrm{g}$ cell or tissue extracts) were resolved by SDS-PAGE under reducing conditions and subjected to Western blot analysis. NRP-1 was detected using rabbit polyclonal antibody (OriGene). After transfer to a PVDF membrane, Western blots were developed by ECL following the Vendor protocol (Pierce, Rockford, IL). The bands were quantified using Quantity One V4.52 software (Bio-Rad Laboratories).

Statistics. Unsupervised hierarchical clustering was performed on microarray data. The statistical significance of differentially expressed miRNAs obtained from microarray data was analyzed by the Welch two-sample t-test with a P-value $<0.05$. Statistical differences were evaluated using non-parametric tests, including the Mann-Whitney $U$ test for differences between two groups and the Kruskall-Wallis test for differences between three or more groups. Statistical differences were also evaluated using the Student's t-test (two-tailed) between two groups and P-values $<0.05$ were considered significant. All calculations were performed using SPSS version 13.0.

\section{Results}

Microarray detection of miRNAs differentially expressed in primary CRC and liver metastasis tissues. We compared miRNA expression in three matched pairs of primary carcinoma and liver metastasis tissues by hybridization of miRNAs onto microarrays. Only miRNAs that were altered by at least 1.5-fold between primary CRC and liver metastasis were chosen for further analysis. We identified 19 upregulated miRNAs and 18 downregulated miRNAs between primary CRC and liver metastasis tissues. Using the Welch two-sample t-test, 13 miRNAs were found to be significantly different in primary CRC compared to liver metastasis tissues $(\mathrm{P}<0.05$, Table I). Six miRNAs were upregulated and 7 miRNAs were downregulated. Hierarchical clustering of the microarray data generated a tree with a clear miRNA distinction between two major primary and metastatic tissues (Fig. 1).

Determination of the most stable normalizer for real-time $P C R$. Two different algorithms were used to assess the variance of RNA targets in expression levels (GeNorm and NormFinder). Both statistical methods ranked the RNA targets similarly from the most to the least stable, with an excellent correlation in raw stability values (Table II). Both Genorm and NormFinder algorithms report the lowest stability value as the most stable normalizer, whereas the highest values represent the least stable normalizers. In this study, miR-16 was the most consistently expressed miRNA, followed by miR-103, miR-93, miR-23a and miR-191. In contrast, 5S, a commonly used normalizer for miRNA qRT-PCR experiments $(29,30)$, was the least stable. The difference in stability between miR-16 and $5 \mathrm{~S}$ was a standard deviation of nearly $1 \mathrm{Ct}$ (a difference of 2-fold). Table reference gene stability was calculated by Genorm and NormFinder. miRNAs can regulate multiple targets within the 


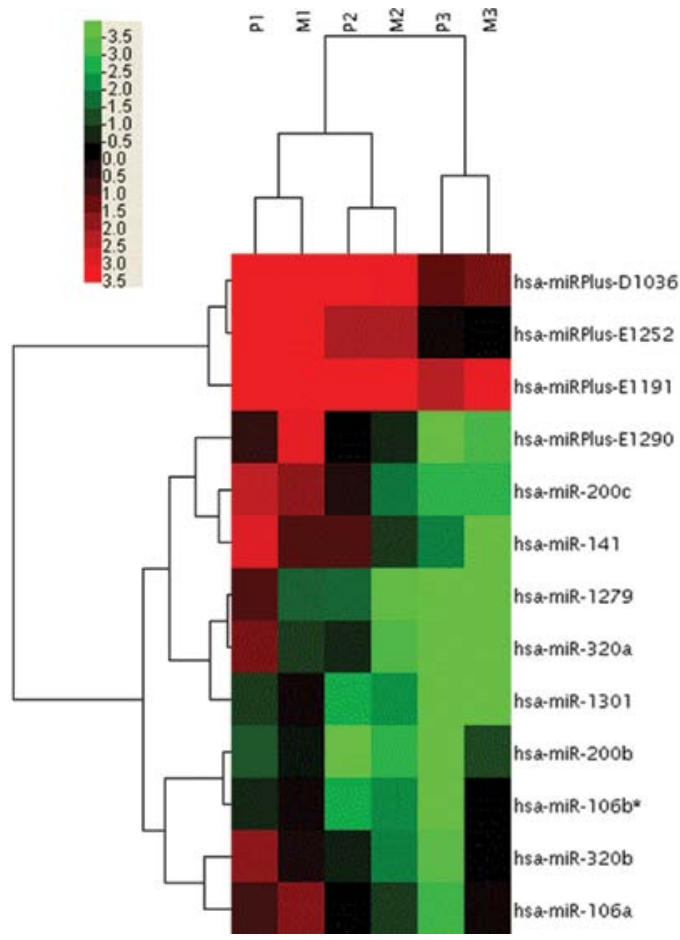

Figure 1. miRNAs are deregulated in primary CRC compared to liver metastasis as detected by microRNA microarray. Three pairs of primary CRC and liver metastasis tissues were analyzed by miRCURY LNA ${ }^{\mathrm{TM}}$ microRNA Arrays v.11.0. Unsupervised hierarchical cluster analysis of miRNA expression in three CRC patients. For each miRNA, red represents higher and green represents lower expression than the average expression. P1, primary CRC, sample 1; M1, liver metastasis, sample 1 .

same pathway and amplify their biological effects; therefore, even relatively small changes in miRNA expression may be biologically significant (31). Thus, rigorous normalization of miRNA data may be even more critical than that of other RNA functional classes for a detailed understanding of miRNA biology.

Validation of microarray data by real-time RT-PCR analysis. To confirm our microarray data, RT-PCR was performed to analyze the expression of the significantly regulated miRNAs,
Table II. The stability values of reference genes by Genorm and Normfinder.

\begin{tabular}{|c|c|}
\hline Reference gene & Stability value \\
\hline \multicolumn{2}{|l|}{ Genorm } \\
\hline hsa-miR-16 & 0.6257 \\
\hline hsa-miR-103 & 0.6692 \\
\hline hsa-miR-93 & 0.7964 \\
\hline hsa-miR-191 & 0.9171 \\
\hline hsa-miR-23a & 0.9171 \\
\hline hsa-miR-24 & 1.1166 \\
\hline hsa-let-7a & 1.1905 \\
\hline hsa-miR-99a & 1.2810 \\
\hline hsa-miR-195 & 1.3845 \\
\hline hsa-miR-17-5p & 1.4881 \\
\hline 5SRNA & 1.5995 \\
\hline \multicolumn{2}{|l|}{ NormFinder } \\
\hline hsa-miR-16 & 0.2727 \\
\hline hsa-miR-24 & 0.3144 \\
\hline hsa-miR-103 & 0.3500 \\
\hline hsa-miR-93 & 0.3722 \\
\hline hsa-miR-195 & 0.4259 \\
\hline hsa-miR-191 & 0.4814 \\
\hline hsa-let-7a & 0.5305 \\
\hline hsa-miR-99a & 0.5893 \\
\hline hsa-miR-195 & 0.7338 \\
\hline hsas-miR-17-5p & 0.7905 \\
\hline 5SRNA & 1.0743 \\
\hline
\end{tabular}

including hsa-miR-320a, -320b, -141, -200c, -200b, -106b and -106a. There are 4 hsa-miRPlus probes designed by the Exiqon Company to predict new miRNAs, and the function of hsa-miR-1279 and -1301 are still unknown. As a result, we did not study these miRNAs. Using 7 primary CRC and 7 corresponding metastasis tissues, qRT-PCR analysis confirmed that the expression levels of miR-320a, $-320 b,-141$, and $-200 c$ were also comparably downregulated (Fig. 2B) and that
A

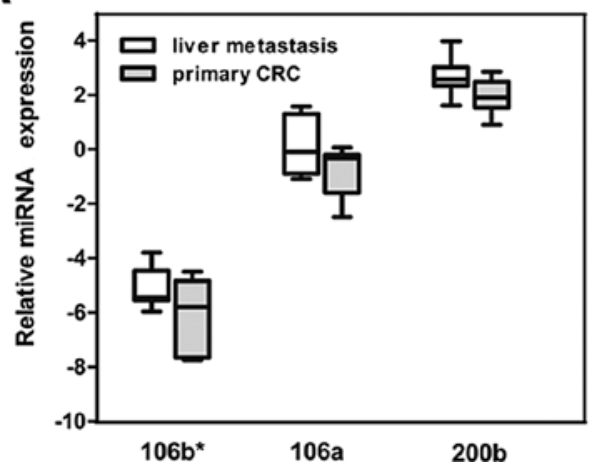

B

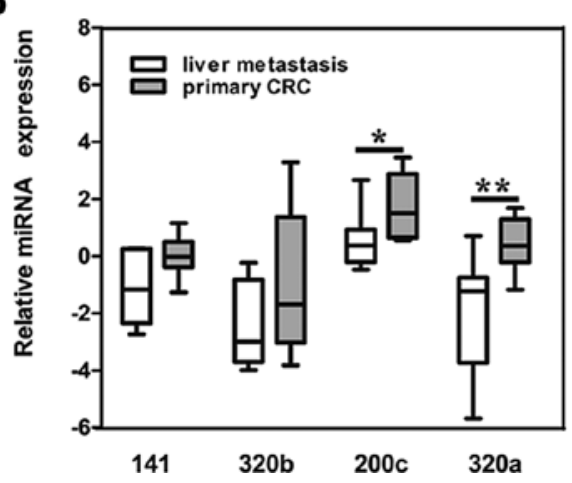

Figure 2. Real-time PCR assays in primary CRC and metastasis. (A) Box and whisker plots of selected miRNAs predicted to be upregulated in liver metastasis. (B) Box and whisker plots of selected miRNAs predicted to be downregulated in liver metastasis. The graph shows the $\log 2$ fold change of the miRNAs. ${ }^{*} \mathrm{p}<0.05,{ }^{* *} \mathrm{p}<0.01$. P-values were calculated using the Mann-Whitney U test. 
Table III. Relationship between clinicopathological factors and expression of miR-320a in tumor tissue.

\begin{tabular}{|c|c|c|c|}
\hline \multirow[b]{2}{*}{ Variables } & \multirow{2}{*}{$\begin{array}{c}\text { No of } \\
\text { patients }\end{array}$} & miR-320a & \multirow[b]{2}{*}{ P-value } \\
\hline & & $($ mean $\pm \mathrm{SEM})$ & \\
\hline Tumor vs. mucosa & & & 0.17890 \\
\hline Colorectal tumor & 62 & $2.045 \pm 0.2104$ & \\
\hline Normal mucosa & 6 & $2.692 \pm 0.5505$ & \\
\hline Clinical stage & & & 0.01870 \\
\hline I & 4 & $2.771 \pm 0.4962$ & \\
\hline II & 21 & $2.605 \pm 0.4668$ & \\
\hline III & 20 & $1.933 \pm 0.3170$ & \\
\hline IV & 17 & $1.318 \pm 0.2336$ & \\
\hline pT category & & & 0.23350 \\
\hline pT2 & 4 & $2.298 \pm 0.7990$ & \\
\hline pT3 & 52 & $2.073 \pm 0.2280$ & \\
\hline pT4 & 6 & $1.663 \pm 0.8116$ & \\
\hline Lymph node & & & 0.06530 \\
\hline Negative & 28 & $2.6 \pm 0.3609$ & \\
\hline Positive & 34 & $1.615 \pm 0.2163$ & \\
\hline Liver metastasis & & & 0.01530 \\
\hline Negative & 45 & $2.335 \pm 0.2580$ & \\
\hline Positive & 17 & $1.268 \pm 0.2466$ & \\
\hline Peritoneal dissemination & & & 0.04645 \\
\hline Absent & 57 & $2.165 \pm 0.2243$ & \\
\hline Present & 5 & $1.039 \pm 0.3901$ & \\
\hline Grade & & & 0.48390 \\
\hline Well & 9 & $1.852 \pm 0.5089$ & \\
\hline Moderate & 37 & $1.928 \pm 0.2668$ & \\
\hline Poor & 15 & $2.403 \pm 0.4579$ & \\
\hline Localization & & & 0.16910 \\
\hline Colon & 43 & $2.235 \pm 0.2783$ & \\
\hline Rectum & 19 & $1.62 \pm 0.2783$ & \\
\hline Tumor diameter (mm) & & & 0.83200 \\
\hline$\leq 50$ & 33 & $2.004 \pm 0.2689$ & \\
\hline$>50$ & 29 & $2.14 \pm 0.3421$ & \\
\hline
\end{tabular}

miR-200b, -106b and -106a were also comparably upregulated (Fig. 2A) between primary CRC and liver metastasis samples; however, the overall expression level of miR-320a and -200c was downregulated significantly in liver metastasis compared to its primary carcinoma, as expected from the microarray data. miR-320a was most significantly downregulated in liver metastasis.

Downregulation of miR-320a is associated with tumor progression and liver metastasis. We postulated that dysregulation of miR-320a may also be associated with clinicopathological features in CRC patients. To test this hypothesis, we used qRT-PCR to analyze miR-320a expression in a large study cohort consisting of 62 patients. The clinical demographics

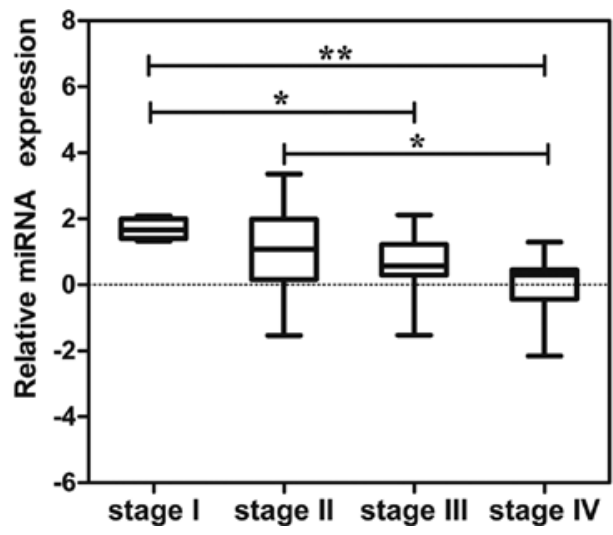

Figure 3. Correlation between clinical stage and expression of miR-320a in patients with CRC. Box and whisker plots of miR-320a which was predicted to correlate with clinical stage of CRC. The graph shows the $\log 2$ fold change of miR-320a. ${ }^{*} \mathrm{P}<0.05,{ }^{* *} \mathrm{P}<0.01$. P-values were calculated using the KruskalWallis test.

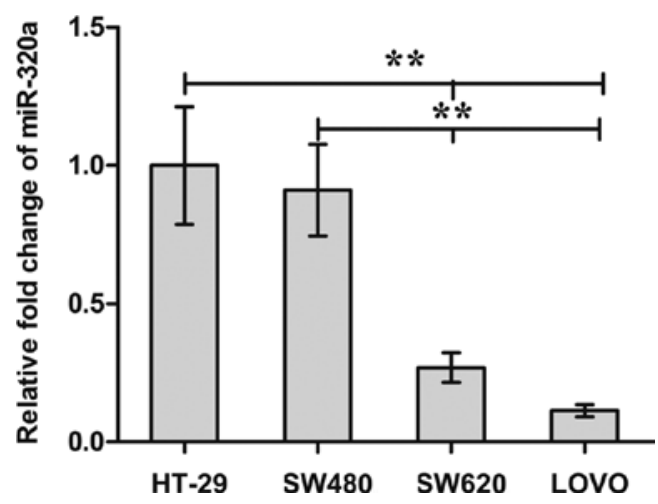

Figure 4. miR-320a expression in colon cancer cell lines by real-time PCR. Student's t-test, $n=3$. $^{* *} \mathrm{P}<0.01$, fold change was calculated by $2^{-\Delta \Delta \mathrm{Ct}}$.

of the study cohort are summarized in Table III. The low expression of miR-320a was associated with an increase in liver metastasis $(\mathrm{P}=0.0153)$ and the development of peritoneal metastases $(\mathrm{P}=0.0464)$ in $\mathrm{CRC}$ patients (Table III). The expression of miR-320a, therefore, was correlated with CRC clinical stage ( $\mathrm{P}=0.0187)$ (Fig. 3, Table III).

miR-320a is highly expressed in metastatic colorectal cancer cells. To identify miRNAs that regulate colorectal cancer metastasis, we selected miR-320a on the basis of clinical specimen research. We investigated the expression of miR-320a in a series of colon cancer cell lines (SW480, HT-29, SW620 and LoVo). SW480 and HT-29 are colon cancer cell lines that possess low metastatic potential, whereas SW620 and LoVo are colon cancer cell lines that show high metastatic potential. The expression of miR-320a was lower in SW620 and LoVo cells while miR-320a expression was elevated in SW480 and HT-29 cells (Fig. 4).

miR-320a does not affect the proliferation of colon cancer cells. To determine whether miR-320a overexpression inhibits colon cancer cell proliferation, we cloned the genomic sequence of the human miR-320a gene into a 
A

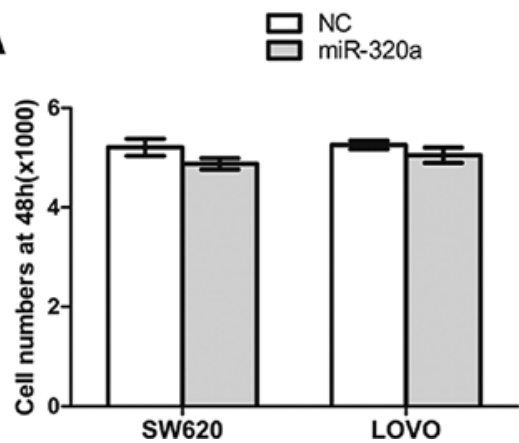

B
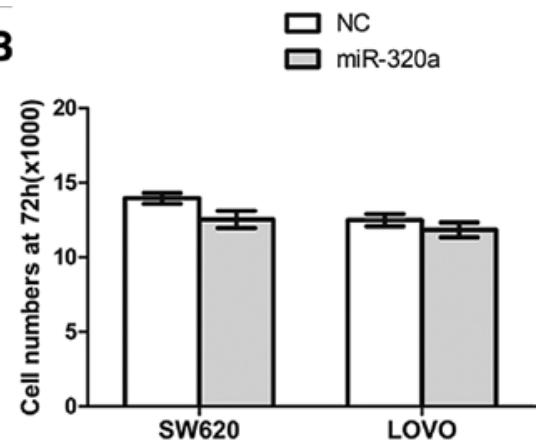

Figure 5. Colon cancer cell proliferation of SW620 and LoVo cells stimulated by miR-320a. (A) Colon cancer cell proliferation stimulated by miR-320a for $48 \mathrm{~h}$; (B) colon cancer cell proliferation stimulated by miR-320a for $72 \mathrm{~h}$.
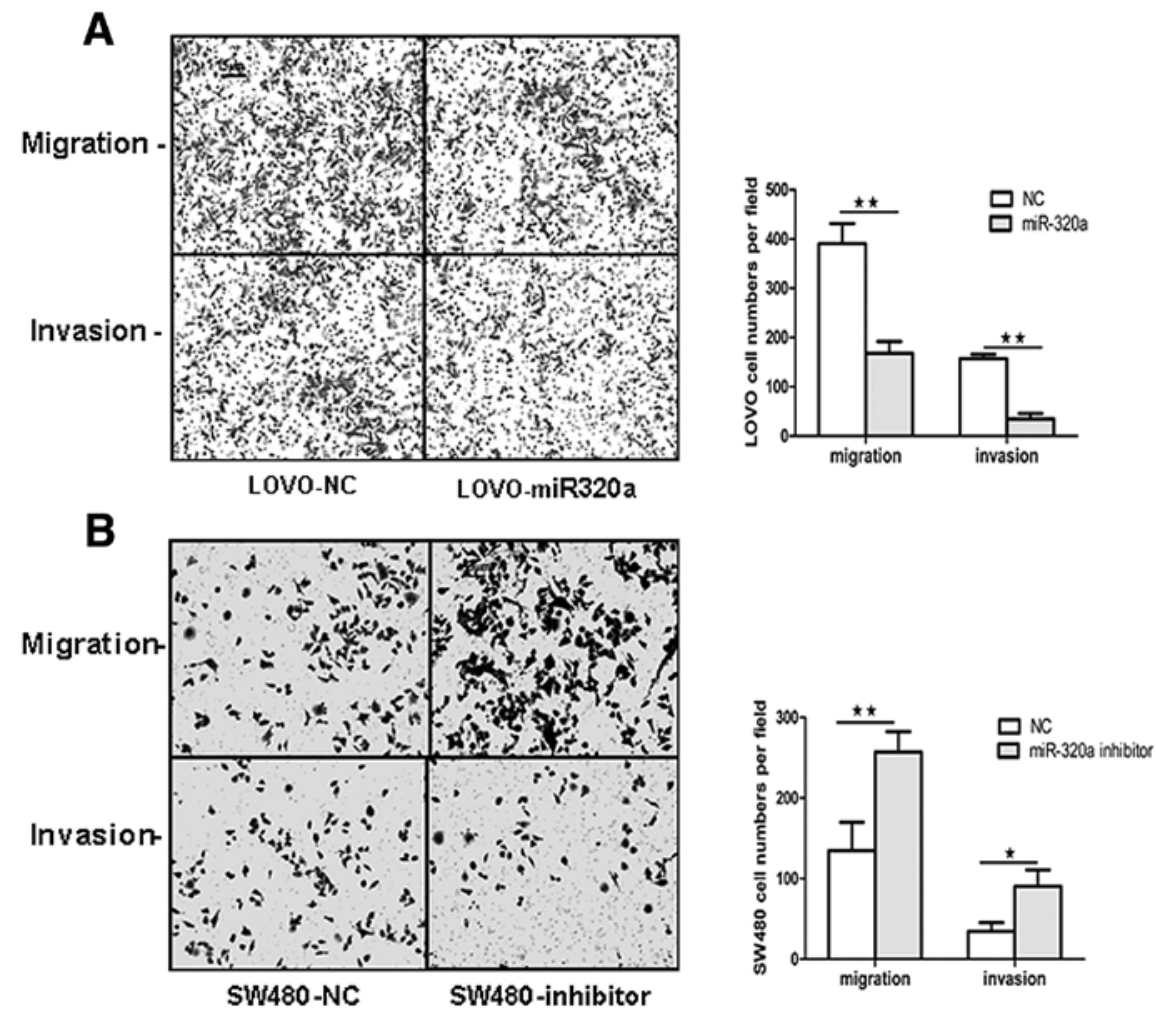

Figure 6. Effect of miR-320 on tumor cell invasion and metastasis in SW480 and LoVo cells. (A) Invasion and migration assays of LoVo cells transfected with miR-320a. Representative fields of migrating (upper panel) or invasive (lower panel) cells on the membrane (left) (original magnification, x100). Average invasive or migrating cell number per field (right). (B) The invasive or migrating cell number of SW480 cells transfected with the miR-320a inhibitor. Representative fields of migrating (upper panel) or invasive (lower panel) cells on the membrane (left) (original magnification, x100). Average invasive or migrating cell number per field (right). Student's t-test; ${ }^{*} \mathrm{P}<0.05,{ }^{* *} \mathrm{P}<0.01 ; \mathrm{n}=10$.

lentivirus vector and packaged this construct into lentivirus. We then used the resulting lentivirus to express miR-320a in SW620 and LoVo cell lines for two to three days. In both cell lines, ectopic expression of miR-320a did not affect cellular proliferation in vitro (Fig. 5).

miR-320a inhibits the migratory and invasive abilities of colon cancer cells in vitro. We investigated the effects of decreased miR-320a expression on cell lines with low metastatic potential and increased miR-320a expression on cell lines with high metastatic potential. We used miR-320a lentivirus- and control lentivirus-infected LoVo cells for these experiments. LoVo cells were derived from the liver metastatic site of a colon cancer patient. Mimic-miR-320a and negative control oligonucleotides were also chemosynthesized and then transfected into SW480 cells for transwell assays in vitro. The overexpression of miR-320a significantly impaired the ability of LoVo cells to migrate and invade through the Matrigelcoated membranes or the non-Matrigel coated membranes towards the serum-containing medium in a modified Boyden chamber assay (Fig. 6A). The depletion of miR-320a significantly increased the migratory and invasive abilities of SW480 cells, as these cells demonstrated a reduced ability to migrate through Matrigel-coated membranes or non-Matrigel-coated 
A

Position 1126-1132 of NRP1 3 ' UTR

5. ... cagganagahacudgcagcudua...

1111111

3. AGCGgGaGAGUUGgGUCGAAAA

hsa-miR-320a

Position 197-203 of NRP1 3' UTR

5. ... GgacucadgugcagucagcuUUD...

3. IIIIIIII

hsa-miR-320a

hsa-miR-320a

Position 1126-1132 of NRP1 3' UTR

UTR mutant $5^{\circ}$... CAGgAAAgAaAcUDGUCUUGACA...

Position 197-203 of NRP1 3' UTR
UTR mutant 5 ' ...GGACUCAUGUGCAGUUCUUGACU...

B

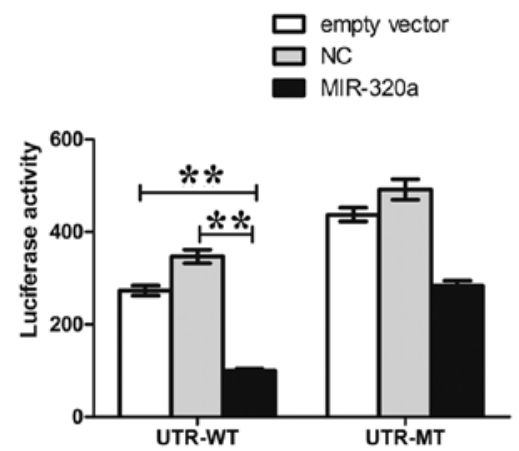

D

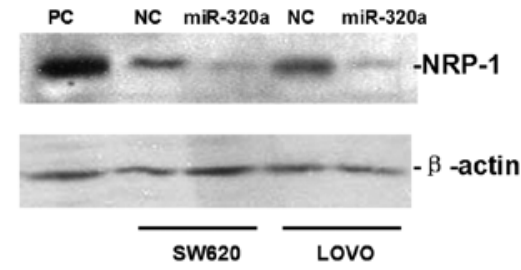

E

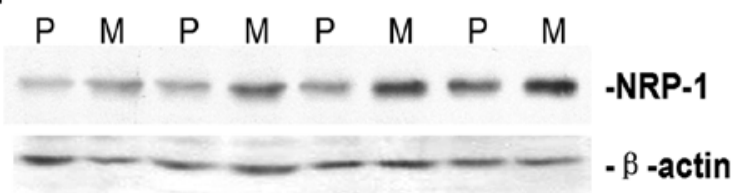

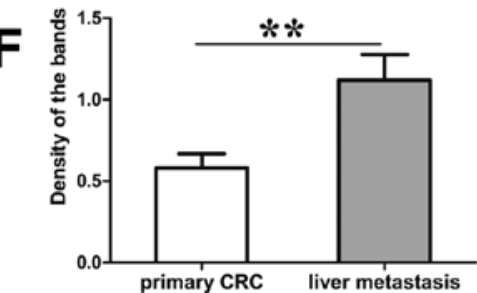

Figure 7. miR-320a directly targets NRP-1. (A) Predicted duplex formation between human NRP-1 3'UTR and human miR-320a. (B) Luciferase activity of wild-type (UTR-WT) or mutant (UTR-MT) NSP-1 3'UTR reporter gene in LoVo cells infected with the miR-320a-expressing or empty vector. (C) Real-time PCR of NSP-1 mRNA expression in LoVo cells infected with the miR-320a-expressing or empty vector. Data were normalized to the level of GAPDH mRNA. (D) Immunoblotting of NSP-1 in LoVo and SW620 cells infected with the miR-320a-expressing or empty vector. (E) NSP-1 protein expression between primary CRC and liver metastasis; (F) Quantification of the bands shown in panel $\mathrm{E}$ after normalization to actin. Student's $\mathrm{t}$-test; ${ }^{* *} \mathrm{P}<0.01, \mathrm{n}=3$.

membranes towards serum-containing medium in a modified Boyden chamber assay (Fig. 6B) when compared with the control cells.

miR-320a downregulates NRP-1 expression by directly targeting its $3^{\prime} U T R$. To understand the mechanisms by which miR-320a induces CRC invasion, we used two computational methods to identify its targets. We focused on miR-320a target genes, with emphasis placed on those genes that were invasion- and metastasis-related. Among the approximately 100 targets predicted by both the TargetScan and PicTar search programs, NRP-1 was of particular interest since its expression has been found to be progressively lost in tumors showing increasing degrees of metastasis. The restored expression of NRP-1 in cancer cells has been found to impair migration and invasion in vitro as well as tumor progression in vivo. The NRP-1-encoded mRNA contains a 3'UTR element that is complementary to miR-320a (Fig. 7A). In an effort to determine whether NRP-1 is regulated by miR-320a through direct binding to its $3^{\prime} \mathrm{UTR}$, we constructed fulllength wild-type and mutant fragments of NRP-1 mRNA 3'UTR and inserted them into the region immediately downstream of a luciferase reporter gene. Subsequently,
pGIF-320a vectors containing different luciferase 3'UTR constructs were co-transfected into LoVo cells. We found that miR-320a decreased the relative luciferase activity of the wild-type 3'UTR of NRP-1, however, luciferase activity did not increase sharply in the UTRs containing mutant binding sites when compared to the mut-type counterparts (Fig. 7B). These data support the idea that NRP-1 is a direct target of miR-320a. Real-time PCR analysis indicated that the expression of NRP-1 in LoVo cells transfected with miR-320a was downregulated compared to those cells transfected with control constructs (Fig. 7C). Western blotting results showed that expression levels of NRP-1 in LoVo cells transfected with pGIF-320a were downregulated compared to those transfected with negative control (Fig. 7D). Western blot analysis also demonstrated that the expression of NRP-1 in liver metastasis was upregulated compared with its primary CRC tissue (Fig. 7E and F).

\section{Discussion}

In this study, we initially analyzed miRNA expression patterns in three pairs of primary tumors and their corresponding liver metastases. Hierarchical cluster analysis of microarray data 
showed that metastases tended to cluster with their corresponding primary tumor rather than with each other. This result is consistent with a clonal development of metastasis from the primary tumor (32). Despite the very similar expression signature between the metastasis and the primary tumor, we found a metastasis-specific miRNA profile characterized by 13 differentially expressed miRNAs (7 upregulated, 6 downregulated). In a cluster analysis, these metastasisspecific miRNAs were able to successfully distinguish all three metastases from the primary carcinoma. Thus, these miRNAs comprise a novel miRNA expression profile for liver metastasis of CRC. Using a larger sample number (seven pairs of metastasis and primary $\mathrm{CRC}$ ), we clearly confirmed that seven miRNAs (miR-106a, -200b, -106b, -200c, -141, -320a and $-320 b$ ) were expressed at comparable levels in the primary tumor and corresponding metastasis; however, only miR-320a and miR-200c were downregulated significantly.

It was recently demonstrated that microRNAs can promote tumor invasion and metastasis in breast cancer, suggesting that the dysregulation of specific miRNAs can provide a selective advantage in breast cancer metastasis. Various miRNAs serve as metastasis activators [miR-10b (33), miR-21 (34,35), miR-9 (36), miR-373 and miR-520c (37)] and are able to initiate invasion and metastasis in breast cancer. Some miRNAs serve as metastasis suppressors [miR-3 (38), miR-200 family $(39,40)$, miR-335 and miR-126 (41)] and are able to inhibit invasion and metastasis in breast cancer. Here, we found some differences between the metastasis-specific miRNAs found in CRC and those found in breast cancer. These findings suggest that the dysregulation of miRNAs in the context of metastasis may depend on the cellular microenvironment.

We found that miR-320a was most significantly downregulated in liver metastasis tissues. The human miR-320 sequence was originally cloned from normal colon mucosa (42), and miR-320 highlights the prognostic potential of miRNAs in relation to stage II colon cancer (43). The human miR-320a gene is located at chromosome $8 \mathrm{p} 21.3$. The region of chromosome 8p21-22 has been identified as a liver metastatic susceptibility locus whose disruption increases metastatic potential (44). Chromosome $8 \mathrm{p}$ is also frequently lost in late stage and metastatic CRC $(45,46)$. Given this, it is likely that a reduction in miR-320a expression may also be associated with clinicopathological parameters in primary CRC. To test this hypothesis, we analyzed miR-320a expression in a large CRC study group. In these tissue specimens, we detected a progressive downregulation of miR-320a that was associated with tumor progression and based on clinical stage and liver metastasis.

In this study, we demonstrated that miR-320a expression in liver metastatic colon cancer cells was downregulated when compared to that in non-metastatic cells. The overexpression of miR-320a in liver metastatic colon cancer cells inhibited their migratory and invasive abilities. Conversely, silencing this miRNA in non-metastatic colon cancer cells allowed them to acquire invasive behavior; however, miR-320a expression levels did not affect the proliferation of these cells.

Our data demonstrate that neuropilin 1 (NRP-1) is a direct target of miR-320a and that miR-320a-mediated suppression of NRP-1 is dependent upon the NRP-1 3'UTR. NRP-1 is a 120 to $130-\mathrm{kDa}$ cell-surface glycoprotein and acts as a co-receptor for vascular endothelial growth factor (VEGF) (47). VEGF has been associated with increased angiogenesis and liver metastasis of colon cancer (48). In preclinical studies, NRP-1 was found to modulate angiogenesis and metastasis of colon cancer. Transfection with NRP-1 led to a greater than 2-fold increase in the migration of KM12SM/LM2 cells (human colon cancer cell line) compared to negative controls. The expression of NRP-1 produced tumors of greater mass, volume, and blood vessel number compared with the same cell line transfected with a control vector following injection into nude mice (49). This indicates that miR-320a is also likely to be involved in regulating certain steps of angiogenesis and metastasis in which NRP-1 is known to participate. These results indicate that miR-320a may inhibit angiogenesis and cancer cell invasion by targeting NRP-1 in CRC. A recent study supports our findings by demonstrating that miR-320 can impair angiogenesis of myocardial microvascular endothelial cells from GK rats (22).

In conclusion, we demonstrated that expression of miR-320a positively correlates with clinical stage and liver metastasis in patients diagnosed with CRC. Our study demonstrates that miR-320a may suppress the invasion and metastasis of CRC by directly binding to the 3'UTR of NRP-1. Although there is still much to learn concerning the role of miR-320a in colorectal tumorigenesis, miR-320a provides us with a novel potential target for the treatment of colon cancer.

\section{Acknowledgements}

This study was supported by the National Natural Science Foundation of China (Grant no. 31140001), Peking University People's Hospital Reasearch and Development Funds (no. RDB2008-04) and a Doctoral Fund from the Ministry of Education of China (no. 20090001120084).

\section{References}

1. Espey DK, Wu XC, Swan J, et al: Annual report to the nation on the status of cancer, 1975-2004, featuring cancer in American Indians and Alaska Natives. Cancer 110: 2119-2152, 2007.

2. Ferlay J, Autier P, Boniol M, Heanue M, Colombet $M$ and Boyle P: Estimates of the cancer incidence and mortality in Europe in 2006. Ann Oncol 18: 581-592, 2007.

3. Weiss L, Grundmann E, Torhorst J, et al: Haematogenous metastatic patterns in colonic carcinoma: an analysis of 1541 necropsies. J Pathol 150: 195-203, 1986.

4. Rudmik LR and Magliocco AM: Molecular mechanisms of hepatic metastasis in colorectal cancer. J Surg Oncol 92: 347-359, 2005.

5. Bird NC, Mangnall D and Majeed AW: Biology of colorectal liver metastases: a review. J Surg Oncol 94: 68-80, 2006.

6. Takayama T, Miyanishi K, Hayashi T, Sato Y and Niitsu Y: Colorectal cancer: genetics of development and metastasis. J Gastroenterol 41: 185-192, 2006.

7. Nadal C, Maurel $J$ and Gascon P: Is there a genetic signature for liver metastasis in colorectal cancer? World J Gastroenterol 13: 5832-5844, 2007.

8. Bartel DP: MicroRNAs: target recognition and regulatory functions. Cell 136: 215-233, 2009.

9. Lewis BP, Burge CB and Bartel DP: Conserved seed pairing, often flanked by adenosines, indicates that thousands of human genes are microRNA targets. Cell 120: 15-20, 2005.

10. Calin GA, Sevignani C, Dumitru CD, et al: Human microRNA genes are frequently located at fragile sites and genomic regions involved in cancers. Proc Natl Acad Sci USA 101: 2999-3004, 2004.

11. He L, Thomson JM, Hemann MT, et al: A microRNA polycistron as a potential human oncogene. Nature 435: 828-833, 2005. 
12. Johnson SM, Grosshans H, Shingara J, et al: RAS is regulated by the let-7 microRNA family. Cell 120: 635-647, 2005.

13. Lu J, Getz G, Miska EA, et al: microRNA expression profiles classify human cancers. Nature 435: 834-838, 2005.

14. Roldo C, Missiaglia E, Hagan JP, et al: microRNA expression abnormalities in pancreatic endocrine and acinar tumors are associated with distinctive pathologic features and clinical behavior. J Clin Oncol 24: 4677-4684, 2006.

15. Slaby O, Svoboda M, Michalek J and Vyzula R: microRNAs in colorectal cancer: translation of molecular biology into clinical application. Mol Cancer 8: 102, 2009.

16. Farazi TA, Spitzer JI, Morozov P and Tuschl T: miRNAs in human cancer. J Pathol 223: 102-115, 2011.

17. Nicoloso MS, Spizzo R, Shimizu M, Rossi S and Calin GA: microRNAs - the micro steering wheel of tumour metastases. Nat Rev Cancer 9: 293-302, 2009.

18. Kulda V, Pesta M, Topolcan O, et al: Relevance of miR-21 and miR-143 expression in tissue samples of colorectal carcinoma and its liver metastases. Cancer Genet Cytogenet 200: 154-160, 2010.

19. Baffa R, Fassan M, Volinia S, et al: microRNA expression profiling of human metastatic cancers identifies cancer gene targets. J Pathol 219: 214-221, 2009.

20. Yan LX, Huang XF, Shao Q, et al: microRNA miR-21 overexpression in human breast cancer is associated with advanced clinical stage, lymph node metastasis and patient poor prognosis. RNA 14: 2348-2360, 2008.

21. Schaar DG, Medina DJ, Moore DF, Strair RK and Ting Y: miR-320 targets transferrin receptor 1 (CD71) and inhibits cell proliferation. Exp Hematol 37: 245-255, 2009.

22. Wang XH, Qian RZ, Zhang W, Chen SF, Jin HM and Hu RM: MicroRNA-320 expression in myocardial microvascular endothelial cells and its relationship with insulin-like growth factor-1 in type 2 diabetic rats. Clin Exp Pharmacol Physiol 36: 181-188, 2009.

23. He X, Zhang Q, Liu Y and Pan X: Cloning and identification of novel microRNAs from rat hippocampus. Acta Biochim Biophys Sin (Shanghai) 39: 708-714, 2007.

24. He XJ, Zhang Q, Liu YJ and Pan XY: Increasing specificity of real time PCR to detect microRNA through primer design and annealing temperature increase. Beijing Da Xue Xue Bao 41 691-698, 2009 (In Chinese).

25. Shi R and Chiang VL: Facile means for quantifying microRNA expression by real-time PCR. Biotechniques 39: 519-525, 2005.

26. Peltier HJ and Latham GJ: Normalization of microRNA expression levels in quantitative RT-PCR assays: identification of suitable reference RNA targets in normal and cancerous human solid tissues. RNA 14: 844-852, 2008.

27. Vandesompele J, De Preter K, Pattyn F, et al: Accurate normalization of real-time quantitative RT-PCR data by geometric averaging of multiple internal control genes. Genome Biol 3 . Research0034, 2002.

28. Andersen CL, Jensen JL and Orntoft TF: Normalization of real-time quantitative reverse transcription-PCR data: a modelbased variance estimation approach to identify genes suited for normalization, applied to bladder and colon cancer data sets. Cancer Res 64: 5245-5250, 2004.

29. Takamizawa J, Konishi H, Yanagisawa K, et al: Reduced expression of the let-7 microRNAs in human lung cancers in association with shortened postoperative survival. Cancer Res 64: 3753-3756, 2004.

30. Pineles BL, Romero R, Montenegro D, et al: Distinct subsets of microRNAs are expressed differentially in the human placentas of patients with preeclampsia. Am J Obstet Gynecol 196: 261e1-6, 2007.
31. Calin GA and Croce CM: microRNA - cancer connection: the beginning of a new tale. Cancer Res 66: 7390-7394, 2006.

32. Fidler IJ: The pathogenesis of cancer metastasis: the 'seed and soil' hypothesis revisited. Nat Rev Cancer 3: 453-458, 2003.

33. Ma L, Teruya-Feldstein J and Weinberg RA: Tumour invasion and metastasis initiated by microRNA-10b in breast cancer. Nature 449: 682-688, 2007.

34. Zhu S, Wu H, Wu F, Nie D, Sheng S and Mo YY: microRNA-21 targets tumor suppressor genes in invasion and metastasis. Cell Res 18: 350-359, 2008 .

35. Song B, Wang C, Liu J, et al: MicroRNA-21 regulates breast cancer invasion partly by targeting tissue inhibitor of metalloproteinase 3 expression. J Exp Clin Cancer Res 29: 29, 2010.

36. Ma L, Young J,Prabhala H, et al: miR-9, a MYC/MYCN-activated microRNA, regulates E-cadherin and cancer metastasis. Nat Cell Biol 12: 247-256, 2010.

37. Huang Q, Gumireddy K, Schrier M, et al: The microRNAs miR-373 and miR-520c promote tumour invasion and metastasis. Nat Cell Biol 10: 202-210, 2008.

38. Valastyan S, Reinhardt F, Benaich $\mathrm{N}$, et al: A pleiotropically acting microRNA, miR-31, inhibits breast cancer metastasis. Cell 137: 1032-1046, 2009.

39. Burk U, Schubert J, Wellner U, et al: A reciprocal repression between ZEB1 and members of the miR-200 family promotes EMT and invasion in cancer cells. EMBO Rep 9: 582-589, 2008.

40. Korpal M,Lee ES,Hu G and Kang Y: The miR-200 family inhibits epithelial-mesenchymal transition and cancer cell migration by direct targeting of E-cadherin transcriptional repressors ZEB1 and ZEB2. J Biol Chem 283: 14910-14914, 2008.

41. Tavazoie SF, Alarcon C, Oskarsson T, et al: Endogenous human microRNAs that suppress breast cancer metastasis. Nature 451: 147-152, 2008.

42. Michael MZ, SM OC, van Holst Pellekaan NG, Young GP and James RJ: Reduced accumulation of specific microRNAs in colorectal neoplasia. Mol Cancer Res 1: 882-891, 2003.

43. Schepeler T, Reinert JT, Ostenfeld MS, et al: Diagnostic and prognostic microRNAs in stage II colon cancer. Cancer Res 68: 6416-6424, 2008

44. Knosel T, Schluns K, Stein U, et al: Chromosomal alterations during lymphatic and liver metastasis formation of colorectal cancer. Neoplasia 6: 23-28, 2004.

45. Blaker H, Graf M, Rieker RJ and Otto HF: Comparison of losses of heterozygosity and replication errors in primary colorectal carcinomas and corresponding liver metastases. J Pathol 188: 258-262, 1999

46. Macartney-Coxson DP, Hood KA, Shi HJ, et al: Metastatic susceptibility locus, an $8 \mathrm{p}$ hot-spot for tumour progression disrupted in colorectal liver metastases: 13 candidate genes examined at the DNA, mRNA and protein level. BMC Cancer 8: $187,2008$.

47. Soker S, Takashima S, Miao HQ, Neufeld G and Klagsbrun M: Neuropilin-1 is expressed by endothelial and tumor cells as an isoform-specific receptor for vascular endothelial growth factor. Cell 92: 735-745, 1998.

48. Neal CP, Garcea G, Doucas H, et al: Molecular prognostic markers in resectable colorectal liver metastases: a systematic review. Eur J Cancer 42: 1728-1743, 2006.

49. Parikh AA, Fan F, Liu WB, et al: Neuropilin-1 in human colon cancer: expression, regulation, and role in induction of angiogenesis. Am J Pathol 164: 2139-2151, 2004. 